Aim of the study: Gastrointestinal lymphoma is the most common type of extranodal lymphoma and commonly involved site is the stomach. We have compared the superiority between treatment modalities for primary gastric lymphoma and we want to investigate efficacy of rituximab in gastric lymphoma.

Material and methods: Between April 2002 and December 2011, 146 patients with a histologically confirmed primary gastric lymphoma, initially diagnosed at eight different Cancer Centers within Turkey were evaluated retrospectively. According to the treatment modality, the patients were divided into chemotherapy (CT) alone, chemotherapy and radiotherapy (CRT), surgery and chemotherapy (SCT), surgery along with chemotherapy and radiotherapy (SCRT), and surgery (S) alone groups.

Results: Median follow-up period was 25.5 months. The 5-year EFS (event free survival) and OS (overall survival) rates for the patients were $55 \%$ and $62.3 \%$ respectively. In Log rank analysis of OS and EFS, we have identified levels of albumin and hemoglobine, IPI score, stage at diagnosis as factors influencing survival. In multivariate analysis of OS and EFS, only albumin and stage at diagnosis were factors independently contributing to survival. There was no statistically significant difference in terms of survival between different treatment modalities $(p=0.707$ in EFS and $p=$ $=0.124$ in OS). In analysis of patients treated with chemotherapy alone, there was no a statistically significant difference in terms of EFS and OS between chemotherapy regimens with or without rituximab in localized and advanced stage groups ( $p=0.264$ and $p=0.639$ ). There was no statistical difference in survival rate (EFS and OS) between surgical or non-surgical treatment modalities for localized/ advanced stage gastric lymphoma groups ( $p=0.519 / p=0.165)$.

Conclusions: There are several treatment options due to similar results in different treatment modalities. Also benefit of rituximab treatment in gastric lymphoma is still a controversial subject. Additional prospective trials are definitely required in order to clarify use of rituximab in treatment of extranodal gastric lymphoma.

Key words: lymphoma, rituximab, prognosis.

Contemp Oncol (Pozn) 2014; 18 (4): 273-278 DOI: $10.5114 /$ wo.2014.40556

\section{Treatment modalities in primary gastric lymphoma: the effect of rituximab and surgical treatment. A study by the Anatolian Society of Medical Oncology}

Kucukoner Mehmet ${ }^{1}$, Cihan Sener ${ }^{2}$, Ummugul Uyeturk ${ }^{3}$, Mesut Seker ${ }^{4}$, Didem Tastekin ${ }^{5}$, Onder Tonyali ${ }^{6}$, Ozan Balakan ${ }^{7}$, Omer Kemal Yazici, Zuhat Urakci ${ }^{1}$, Abdurrahman Isikdogan ${ }^{1}$, Nuriye Ozdemir ${ }^{2}$, Ali Inal ${ }^{1}$, Muhammed Ali Kaplan ${ }^{1}$, Ali Suner ${ }^{7}$, Sinan Dal ${ }^{8}$, Dogan Uncu², Mahmut Gumus ${ }^{4}$, Melih Cem Boruban ${ }^{5}$, Berna Oksuzoglu³ ${ }^{3}$, Orhan Ayyildiz ${ }^{8}$, Mustafa Benekli ${ }^{6}$

${ }^{1}$ Dicle University, Medical Oncology Department, Turkey

${ }^{2}$ Ankara, Medical Oncology Department, Turkey

${ }^{3}$ Ankara Oncology Hospital, Turkey

${ }^{4}$ Medical Oncology Department, Kartal Hospital, Turkey

${ }^{5}$ Medical Oncology Department, Selcuk University, Turkey

${ }^{6}$ Medical Oncology Department, Gazi University, Turkey

${ }^{7}$ Medical Oncology Department, Gaziantep University, Turkey

${ }^{8}$ Haematology Department, Dicle University, Turkey

\section{Introduction}

Primary gastric lymphoma ( $\mathrm{PGL}$ ) originates in the stomach and may or may not involve the peri-gastric and/or abdominal lymph nodes. The most common site of primary extra nodal non-Hodgkin's lymphoma ( $\mathrm{NHL})$ is the stomach [1, 2]. Diffuse large B-cell lymphoma (DLBCL) is the most common histological subtype [3]. These tumour cells are known to be CD20 positive. Rituximab is an anti-CD20 antibody and is highly effective in nodal DLBCL. Overall 5 -year survival between $50 \%$ and $70 \%$ is reported with multimodality therapy [4].

The modalities of treatment for PGL have been a controversial subject, and the best regimen has not been standardised. The general consensus is local-therapy options (radiotherapy or surgery) and/or systemic chemotherapy according to the stage of the disease. Surgical treatment is usually indicated for gastric lymphomas of localised stage and is performed in patients with advanced stage disease for palliative reasons only. While surgery was the primary mode of treatment in the past, today some studies suggest the use of gastric protective treatments with chemotherapy and radiotherapy instead of surgical primary treatments in lymphoma of the stomach [5]. Rituximab is an anti-CD20 monoclonal antibody and is a highly effective and well-tolerated therapy in nodal DLBCL in combination with chemotherapy [6]. However, the efficacy of rituximab in the treatment of gastric DLBCL is only weakly supported by the published literature, and an increasing number of studies have reported that rituximab does not contribute significantly to the treatment of gastric DLBCL. For example, outcomes in early-stage PGL were not improved by the addition of rituximab to CHOP chemotherapy [7]. Therefore, the role of rituximab in gastric DLBCL remains controversial.

In general, there is no consensus on the optimal treatment modality for $P G L$. To resolve these questions we investigated the efficacy of surgery and rituximab in primary gastric lymphoma. 


\section{Material and methods}

\section{Patients, clinical features and stage}

This retrospective study included 146 Primary Gastric DLBL patients initially diagnosed at eight different Cancer Centres within Turkey from April 2002 to December 2011. Each of the cases complied with the PGL diagnosis criteria as defined by the World Health Organisation (WHO) classification and criteria as defined in other studies [8]. Performance status and serum concentration of lactate dehydrogenase (LDH), as well as serum albumin and haemoglobin levels, were laboratory and clinical features evaluated for potential prognostic importance. We defined low haemoglobin as $<12 \mathrm{~g} / \mathrm{dl}$, low albumin as $<3.5 \mathrm{~g} / \mathrm{dl}$ and high LDH as $>245 \mathrm{U} /$ l. Haematoxylin and eosin stain was used to evaluate the presence or absence of Helicobacter pylori on formalin-fixed, paraffin-embedded specimens.

Disease stage was designated in all patients according to the Lugano staging system for gastrointestinal NHL [9].

\section{Treatment, response criteria and international prognostic index (IPI)}

Treatment modalities such as chemotherapy (CT) alone, chemotherapy and radiotherapy (CRT), surgery and chemotherapy (SCT), surgery along with chemotherapy and radiotherapy (SCRT), and surgery (S) alone were performed. Chemotherapy was applied to 140 patients in the study, and the CHOP schedule was applied to all of the patients in the chemotherapy regime. Rituximab was applied in 109 patients undergoing CHOP chemotherapy. The CHOP schedule was applied as follows: $750 \mathrm{mg} / \mathrm{m}^{2}$ cyclophosphamide on day 1 , $50 \mathrm{mg} / \mathrm{m}^{2}$ doxorubicin on day $1,1.4 \mathrm{mg} / \mathrm{m}^{2}$ vincristine on day 1 , and $100 \mathrm{mg} / \mathrm{d}$ prednisone for 5 days, every 21 days. Rituximab $\left(375 \mathrm{mg} / \mathrm{m}^{2}\right)$ was administrated to patients on day 1 of each CHOP cycle. Radiotherapy was delivered at doses of 40 Gy to the abdomen and gastric bed. covers primary tumour as well as regional lymph nodes.

We defined response criteria according to the International Working Group Recommendations [10]. Complete remission (CR) was defined as the complete disappearance of radiological and physical evidence following treatment. The criteria of the International Non-Hodgkin's Lymphoma Prognostic Factor Project were used to assess patient prognosis [11]. Risk factor variables in the IPI (International Prognostic Index) were age $>60$ years, stage III-IV disease, performance status $\geq 2$, elevated serum LDH level, and number of extra nodal disease sites greater than one. The total sum of the number of risk factors present at diagnosis was used to determine risk groups. The resulting three risk groups were as follows: low risk group (0-1 risk factor), low-intermediate risk group (at least 2 risk factors), and high-intermediate and high-risk group (> 3 risk factors). Patients were separated into two groups, low-intermediate and high-risk, according to the IPI risk situation.

Toxicity was graded from 1 to 4 according to the National Cancer Institute Common Toxicity Criteria (version 2.0) [12].

\section{Statistical analysis}

Event-free survival (EFS) was calculated from the date of diagnosis to the date of treatment failure, disease re- currence or death as a result of lymphoma or acute toxicity of treatment. Overall survival (OS) was measured from the date of diagnosis until the date of death, due to any cause, or the date of final follow-up in survivors.

Kaplan-Meier survival estimates were calculated. Univariate analyses were performed using the log-rank test. The $\chi^{2}$ test, or Fisher's exact test used when the $\chi^{2}$ test assumptions did not apply due to low expected cell counts, were used to compare proportions within different groups. All statistical analysis was completed using SPSS 18.0 for Windows.

\section{Results}

\section{Patient characteristics and survival/prognostic} analysis

The overall characteristics of the 146 gastric DLBCL patients are summarised in Table 1 . Median follow-up period was 25.5 months (range 2.2-120.1 months) and $3-5$-year OS rates were $68.7 \%$ and $62.3 \%$, respectively. The 3-5-year EFS rates were $64.3 \%$ and $55 \%$. Five-year overall survival rates according to the main characteristics of the patients are summarised in Table 1 . During the follow-up period, 46 (31.5\%) patients died and 52 (35.6\%) of the patients had a relapse. Significant differences in terms of survival according to age group, performance status, stage, IPI risk group, serum albumin level, and haemoglobin level were revealed in log-rank analysis. Patients aged $\leq 60$ years had a significantly increased rate of survival (EFS and OS) than patients aged $>60$ years $(p=0.002$ and $p<0.001)$. Significantly better survival was observed in patients with low and intermediate IPI ( $p=0.001$ and $p<0.001$, respectively) than in patients with a high IPI risk score. Similarly, EFS and OS rates were significantly different in patients with low performance scores (EFS and OS) ( $p=0.001$ and $p<0.001$ ), and with early-stage disease (stage I and II) (EFS and OS) ( $p=0.023$ and $p<0.001)$. Conversely, low haemoglobin $(p=0.004$ and $p=0.036)$ and albumin $(p=0.029$ and $p=0.028)$ resulted in worse survival rates (Table 1). Multivariate analysis of the characteristics of the patients is summarised in Table 2. Low albumin [HR = $=0.095(0.019-0.474), p=0.004]$, and stage IV disease $[H R=4.098$ (1.082-15.517), $p=0.038]$ were independently significant factors in multivariate analysis of OS, and stage IV disease [HR = $3.040(1.004-9.208), p=0.049]$ and low albumin [HR $=0.246(0.070-0.865), p=0.029]$, were independently significant factors in multivariate analysis of EFS.

\section{Treatment}

The treatment strategies are summarised in Table 1. There is no statistical difference in survival rate (EFS and OS) among treatment modalities ( $p=0.707$ and $p=0.124$ ) (Fig. 1). Surgical treatment modalities (S, SCT and SCRT) and non-surgical treatment modalities (CT and CRT) were examined in the two groups. In the subgroup analysis, there was no statistical difference in survival rates (EFS and OS) between surgical or non-surgical treatment modalities for patient groups who had localised stage I and 
Table 1. Univariate analysis of variables with influence on survival and relapse

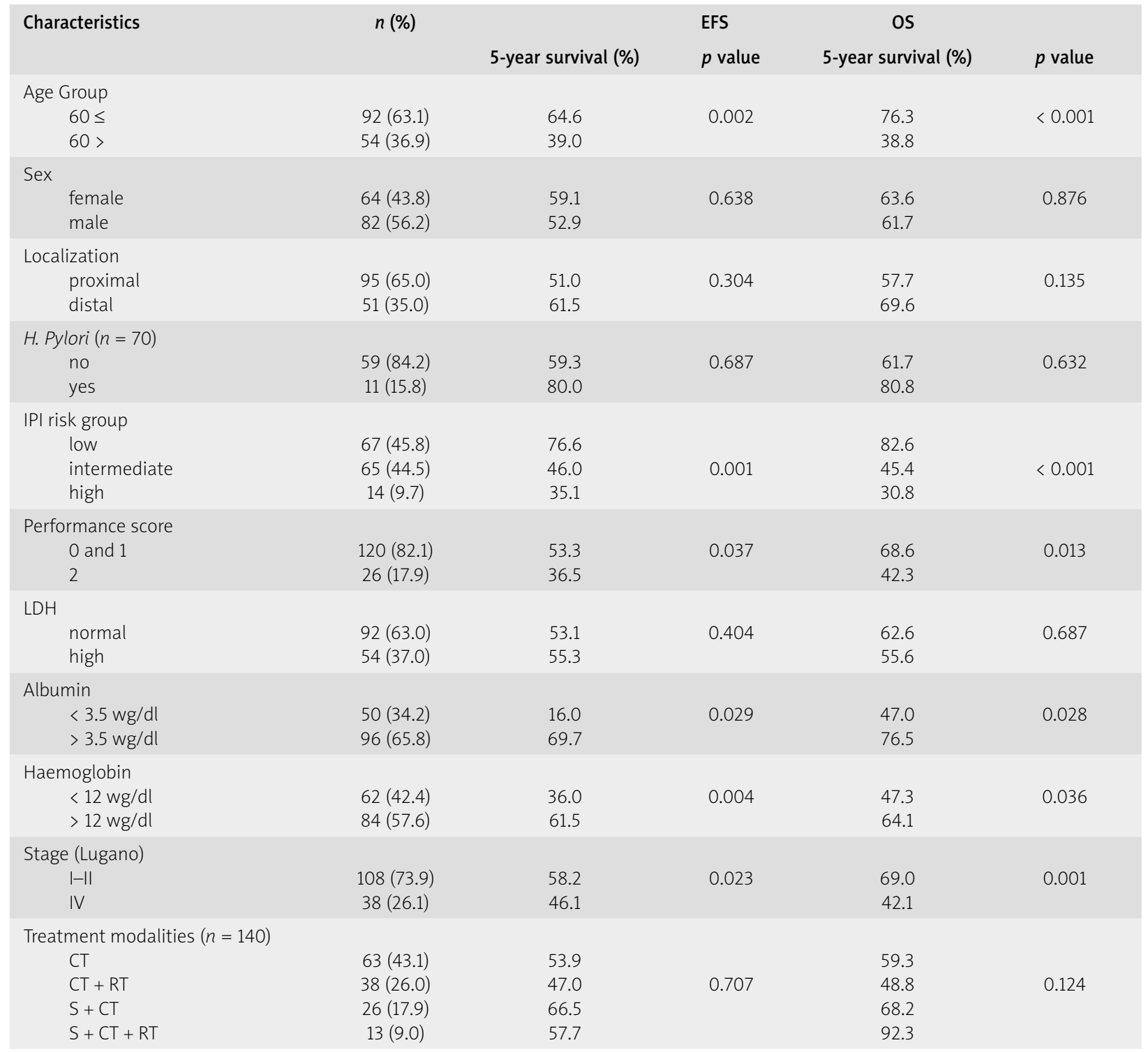

CT - chemotherapy; $R T$ - radiotherapy; S - surgery; IPI - International Prognostic Index

II gastric lymphoma ( $p=0.519$ and $p=0.165$, respectively) (Table 3).

There was no significant relationship in chi-squared analysis of chemotherapy with and without rituximab in all patients $(p=0.068)$. Complete response rates were increased among patients who used the treatment regimen without rituximab. There was a complete response for 73 (67\%) of the 109 patients who were treated with rituximab, while there was a complete response for 26 $(83.9 \%)$ of the 31 patients who were treated without rituximab. $\chi^{2}$ analysis revealed no statistical difference between treatment with rituximab and the response to treatment for patient groups who received CT and CRT ( $p=$ $=0.602$ and $p=0.385$, respectively). There was no statistical difference in EFS and OS between chemotherapies with rituximab and without rituximab for a group (CT) of 63 patients ( $p=0.264$ and $p=0.639$, respectively) (Table 4).
Also, the addition of rituximab to treatment did not result in statistically significant differences in EFS or OS for the group (CRT) of 38 patients who were given both chemotherapy and radiotherapy ( $p=0.501$ and $p=0.692$ ). There was no statistical difference between patients treated with or without rituximab among patients who were at early disease stage (stage I-II) and advanced stage (stage IV) in terms of EFS and OS.

The median number of cycles of chemotherapy was 6 (1-8) in patients who had chemotherapy. Chemotherapy standard cycle 6-8 was not given to 14 of the 140 patients who had chemotherapy for toxicity. Haematological (granulocytopaenia etc.) and gastrointestinal (nausea and vomiting etc.) toxicities were the most common. In general, we observed grade 1-2 haematological and gastrointestinal toxicities in 27 (18.5\%) and 42 patients (28.8\%), respectively. Grade 3-4 gastrointestinal and hae- 
Table 2. Multivariate analysis of variables with influence on survival and relapse

\begin{tabular}{|c|c|c|c|c|}
\hline \multirow[t]{2}{*}{ Characteristic } & \multicolumn{2}{|c|}{ Influence on relapse } & \multicolumn{2}{|c|}{ Influence on survival } \\
\hline & hazard ratio $(95 \% \mathrm{Cl})$ & $p$ value & hazard ratio $(95 \% \mathrm{Cl})$ & $p$ value \\
\hline Stage (IV/I-II) & 3.040 (1.004-9.208) & 0.049 & 4.098 (1.082-15.517) & 0.038 \\
\hline IPI (high/low-interm.) & $0.348(0.040-2.989)$ & 0.336 & $0.541(0.060-4.864)$ & 0.584 \\
\hline Surgery (no/yes) & $0.750(0.257-2.191)$ & 0.599 & $0.351(0.075-1.651)$ & 0.185 \\
\hline $\begin{array}{l}\text { Haemoglobin group } \\
(<12 />12 \mathrm{~g} / \mathrm{dl})\end{array}$ & $0.991(0.308-3.193)$ & 0.988 & $2.215(0.531-9.245)$ & 0.275 \\
\hline $\begin{array}{l}\text { Albumin group } \\
(<3.5 />3.5 \mathrm{~g} / \mathrm{dl})\end{array}$ & $0.246(0.070-0.865)$ & 0.029 & $0.095(0.019-0.474)$ & 0.004 \\
\hline
\end{tabular}

Table 3. Five-year survival rates with surgery and without surgery treatment in localised and advanced stage

$\begin{array}{llcc} & & \begin{array}{c}\text { With surgery treatment } \\ (\mathrm{S}, \mathrm{SCT} \text { and SCRT }) \\ (\mathrm{n}=41), 5 \text {-year survival }(\%)\end{array} & \begin{array}{c}\text { Without surgery treatment } \\ (\mathrm{CT} \text { and CRT) }\end{array} \\ \begin{array}{lll}(\mathrm{n}=102), 5 \text {-year survival }(\%) \\ \text { Stage I-II }\end{array} & \text { EFS } & (n=37), 64 \% & (n=69), 54 \% \\ \text { Stage IV } & \text { EFS } & (n=4), 66 \% & (n=33), 46 \% \\ \text { Stage I-II } & \text { OS } & (n=37), 78 \% & (n=69), 62 \% \\ \text { Stage IV } & \text { OS } & (n=4), 66 \% & (n=33), 41 \%\end{array}$

CT - chemotherapy; RT - radiotherapy; S-surgery; SCT - surgery and chemotherapy; SCRT-surgery along with chemotherapy and radiotherapy

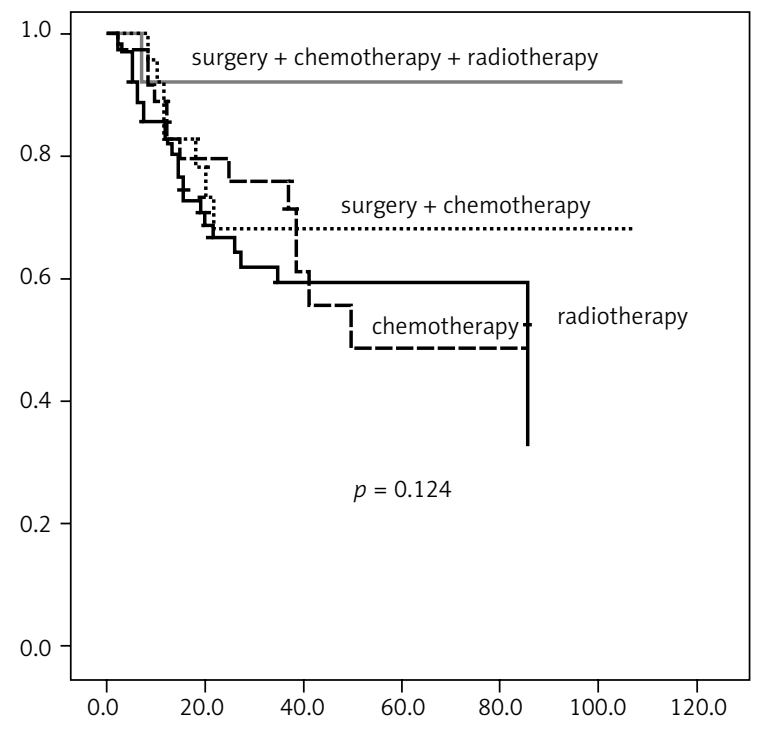

Fig. 1. Comparison of the overall survival curves of patients with different treatment modalitie

matological toxicities occurred in 16 (10.9\%) and 14 (9.5\%) patients, respectively. We observed death-related symptoms (fibril neutropenia, gastrointestinal bleeding) in 8 (5.5\%) patients.

\section{Discussion}

In this study, our aim was to compare the treatment modalities and to investigate the efficacy of surgery, which is one of the treatment modalities applied in primary gastric DLBCL. In particular, we aimed to evaluate the contribution of rituximab, which has already demonstrated its effect in the treatment of nodal DLBCL, to the chemotherapeutic treatment of gastric DLBCL.

According to our results, being under the age of 60 , having low performance scores, being in an early stage (stage $\mathrm{I}$ and II), and having normal albumin and haemoglobin levels resulted in better EFS and OS rates. There was no statistical difference among applied treatment modalities in terms of OS and EFS. However, improved EFS and OS rates were observed in chemotherapy or chemo-radiotherapy treatment modalities that were applied after surgery, although there was no statistical significance in terms of EFS and OS. The log-rank analysis revealed no statistically significant difference in terms of the EFS and OS in patients from the homogeneous subgroups, who received only CT or CRT, in comparison to those who received regimens with rituximab. When the efficacy of rituximab was evaluated in both localised and advanced stage, there was no statistical significance. There was no statistical difference in the $\chi^{2}$ analysis (in the Fisher's exact test) between chemotherapy with rituximab and chemotherapy without rituximab regimens, and response to treatment. There was no relationship between early and advanced stage or between those treated with rituximab and those not.

The results obtained from the studies comparing the treatment modalities in primary gastric lymphoma are summarised below. In a study by Aviles et al., the difference in survival among treatment modalities was compared for early-stage primary gastric DLBCL patients. The best survival rates were seen in the chemotherapy-only group, followed by the combined surgery and chemotherapy group, the group that had undergone surgery alone, and the combined surgery and radiotherapy group $(p<0.001$ for both groups) [7]. Challenging the role of gastrectomy in 
Table 4. Five-year survival rates with rituximab and without rituximab treatment in localised and advanced stage

\begin{tabular}{|c|c|c|c|c|c|c|c|}
\hline & & \multicolumn{2}{|c|}{ Chemotherapy $(n=63)$} & \multirow[t]{2}{*}{$\mathrm{p}$} & \multicolumn{2}{|c|}{ Chemotherapy and radiotherapy $(n=38)$} & \multirow[t]{2}{*}{$\mathrm{p}$} \\
\hline & & $\begin{array}{l}\text { With rituximab } \\
\qquad(\mathrm{n}=50)\end{array}$ & $\begin{array}{l}\text { Without rituximab } \\
\qquad(\mathrm{n}=13)\end{array}$ & & $\begin{array}{l}\text { With rituximab } \\
\qquad(\mathrm{n}=31)\end{array}$ & $\begin{array}{l}\text { Without rituximab } \\
\qquad(\mathrm{n}=7)\end{array}$ & \\
\hline & & $(n=31)$ & $(n=10)$ & & $(n=21)$ & $(n=5)$ & \\
\hline Stage I-II & EFS & $59 \%$ & $57 \%$ & 0.264 & $51 \%$ & $75 \%$ & 0.501 \\
\hline \multirow[t]{2}{*}{ Stage IV } & EFS & $47 \%$ & $100 \%$ & & $42 \%$ & $100 \%$ & \\
\hline & & $(n=19)$ & $(n=3)$ & & $(n=10)$ & $(n=2)$ & \\
\hline Stage I-II & OS & $57 \%$ & $44 \%$ & 0.639 & $61 \%$ & $75 \%$ & 0.692 \\
\hline Stage IV & OS & $54 \%$ & $33 \%$ & & $39 \%$ & $50 \%$ & \\
\hline
\end{tabular}

the management of patients with PGL, comparable survival results were obtained using combination chemotherapy, occasionally combined with radiation [13]. Additional studies have suggested that conservative non-surgical treatment modalities may achieve equal or even better results in comparison to gastrectomy [14]. Recently, the importance of stomach-conserving therapies for treatment of gastric-localised lymphomas has been emphasised [15] In contrast to these studies, there are other, earlier studies that report superior results with surgical treatment modalities. Surgery plays a critical role in the diagnosis, staging and treatment of PGL. Surgical resection followed by chemotherapy has been identified as the treatment of choice in localised gastric lymphoma by several authors [16]. Several earlier studies suggested that gastrectomy significantly improves survival, particularly in stage I and II patients [17]. Surgery plays a controversial role in the management of PGL. Medical treatment of PGL has been gradually replaced by surgery in recent years [18]. When patients treated by chemotherapy alone or with combination therapy (chemotherapy combined with radiation or surgery) are compared, it can be observed that there is no significant difference in survival [19]. In addition, according to another study, in localised gastric DLBCL a similar 5-year survival rate (>90\%) is expected using surgery plus chemotherapy or chemotherapy alone [20]. Compared with these studies, our study showed that there are no differences among the treatment modalities when they were examined separately. In subgroup analysis, in patients who performed surgery with localised or extensive stage, survival benefit was not observed. Surgery does not increase the survival rates in patients with extranodal gastric lymphoma, especially during the localised stage of the disease.

Rituximab treatment was initially introduced for primer nodal lymphoma. A randomised phase III trial by the GELA group highlighted the effectiveness of rituximab treatment of prior nodal B cell lymphoma. Rituximab plus CHOP treatment ( $p<0.001$ and $p=0.007$, respectively) resulted in significantly increased EFS and OS times [6]. The treatment of choice for nodal DLBCL, irrespective of the anatomic site of the lesion, is rituximab plus chemotherapy. Rituximab treatment has been shown to be effective in nodal lymphoma and, therefore, routine usage of rituximab may also be appropriate in the treatment of extra nodal DLBCL. It is not known how the addition of rituximab to chemotherapy regimens effects survival in patients with gastric DLBCL as this has not been tested in a large clinical trial. There are a few studies investigating the contribution of rituximab to treatment of gastric DLBCL; however, all of these involve retrospective studies with the exception of one [19, 21-23]. In the study by Wohrer et al., 15 patients were treated with Rituximab plus chemotherapy. The CR rate was $87 \%$ and the partial response (PR) rate was $13 \%$. As a result, Wohrer concluded that the use of this drug in standard treatment may be premature [22]. It is premature to suggest that rituximab-CHOP be used for standard treatment of patients with localised gastric DLBCL outside of a clinical trial. In other retrospective studies, the 5-year EFS $(p=0.03)$ and OS rates with and without rituximab were $100-100 \%$ vs. $73-63 \%$ [23]. In another study, multivariate analysis for OS revealed the following for treatment without rituximab (HR 2.70 [1.00-7.25]), ( $p=0.049)$ [21]. A phase II study with more patients failed to show any benefit to survival with rituximab treatment. A phase II trial of 42 patients with early-stage disease did not show any advantage of rituximab plus CHOP chemotherapy. In this study, the 5-year EFS and OS were 95\% and 95\%, respectively. There were no statistical differences when compared to historic controls (without rituximab) in the same hospital (In the Aviles study, 42 patients were followed for 3 years and compared with a historic control group) [7]. Also, the results of a phase II study utilising rituximab and CHOP in patients with gastric diffuse large B-cell lymphoma that was started in 2005 with the sponsorship of the company that produces rituximab and finished in 2008, was not published [24]. In two recently published studies, the 3-year EFS rates were $86.0 \%$ for patients receiving $\mathrm{CHOP}$ and $81.7 \%$ for patients receiving R-CHOP. The 3-year OS rates were $94.7 \%$ and $84.7 \%$, respectively ( $p=$ $=0.744$, and $p=0.213$ ) [25]. Five-year EFS and OS rates were not statistically significant. In subgroup analysis, better OS was observed only for patients with advanced-stage disease when rituximab was added [26].

The results of our study pointed out no significant increase in survival by the addition of rituximab to the chemotherapy, and there was no statistically significant difference in survival with or without rituximab chemotherapy in both localised and advanced stage. Because rituximab treatment did not contribute to survival, the addition of rituximab to chemotherapy was discussed more in other recently published studies. the fact that rituximab is not beneficial in extranodal lymphoma might be caused by 
different biological features between nodal lymphomas and extra nodal lymphomas. Still, this point requires further prospective clinical trials. Moreover, in lymphoma treatment, some studies are needed in terms of predictive and prognostic factors [27].

In conclusion, no statistically significant difference was observed in terms of the survival rate among the treatment modalities. Also, the results of our study fail to demonstrate any significant increase in survival by the addition of rituximab to chemotherapy. Because of the different results in the literature, there is still no definitive standard treatment. In particular, the benefit of rituximab treatment in gastric DLBCL is still a controversial subject. Additional prospective trials are required in order to determine the optimal use of rituximab in the treatment of gastric DLBCL.

The authors declare no conflicts of interest.

\section{References}

1. Zucca E, Roggero E, Bertoni F, Conconi A, Cavalli F. Primary extranodal non-Hodgkin's lymphomas. Part 2: Head and neck, central nervous system and other less common sites. Ann Oncol 1999; 10: 1023-33.

2. Al-Akwaa AM, Siddiqui N, Al-Mofleh IA. Primary gastric lymphoma. World J Gastroenterol 2004; 10: 5-11.

3. Koch P, Probst A, Berdel WE, et al. Treatment results in localized primary gastric lymphoma: data of patients registered within the German Multicenter Study (GIT NHL 02/96). J Clin Oncol 2005; 23: 7050-59.

4. Zureikat AH, Heller MT, Zeh HJ. Cancer of the Small Intestine. In Cancer: Principles and Practise of Oncology. Devita VT, Lawrence T, Rosenberg SA (eds.). $9^{\text {th }}$ edition. Lippincott Williams \&Wilkins; Philadelphia 2011; 1055-6.

5. Gobbi PG, Dionigi P, Barbieri F, et al. The role of surgery in the multimodal treatment of primary gastric non-Hodgkin's lymphomas. A report of 76 cases and review of the literature. Cancer 1990; 65: 2528-36.

6. Coiffier B, Lepage E, Briere J, et al. CHOP chemotherapy plus Rituximab compared with $\mathrm{CHOP}$ alone in elderly patients with diffuse large-B-cell lymphoma. N Engl J Med 2002; 346: 235-42.

7. Avilés A, Castañeda C, Cleto S, Neri N, Huerta-Guzmán J, Gonzalez M, Nambo MJ. Rituximab and chemotherapy in primary gastric lymphoma. Cancer Biother Radiopharm 2009; 24: 25-28.

8. Chan JK. The new World Health Organization classification of lymphomas: the past, the present and the future. Hematol Oncol 2001; 19: 129-50.

9. Rohatiner A, d'Amore F, Coiffier B, et al. Report on a workshop convened to discuss the pathological and staging classifications of gastrointestinal tract lymphoma. Ann Oncol 1994; 5: 397-400.

10. Cheson BD, Horning SJ, Coiffier B, Shipp MA, Fisher RI, Connors $J M$, et al. Report of an international workshop to standardize response criteria for non- Hodgkin's lymphomas. NCl Sponsored International Working Group. J Clin Oncol 1999; 17: 1244.

11. The International Non-Hodgkin's Lymphoma Prognostic Factors Project. A predictive model for aggressive non-Hodgkin's lymphoma. N Engl J Med 1993; 329: 987-94.

12. Ajani JA, Welch SR, Raber MN, Fiels WS, Krakoff IM. Comprehensive criteria for assessing therapy-induced toxicity. Cancer Invest 1990; 8: 147-59.

13. Willich NA, Reinartz G, Horst EJ, et al. Operative and conservative management of primary gastric lymphoma: interim results of a German multicenter study. Int I Radiat Oncol Biol Phys 2000; 46: 895-901.
14. Ferreri AJ, Montalban C. Primary diffuse large B-cell lymphoma of the stomach. Crit Rev Oncol Hematol 2007; 63: 65-71.

15. Tondini C, Balzarotti M, Santoro A, et al. Initial chemotherapy for primary resectable large-cell lymphoma of the stomach. Ann Oncol 1997; 8: 497-9.

16. Vaillant JC, Ruskone-Fourmestraux A, Aegerter P, Gayet B, Rambaud JC, Valleur P, Parc R. Management and long-term results of surgery for localized gastric lymphomas. Am J Surg 2000; 179: 216-22.

17. Tedeschi L, Romanelli A, Dallavalle G, Tavani E, Arnoldi E, Vinci M, et al. Stages I and II non-Hodgkin's lymphoma of gastrointestinal tract. Retrospective analysis of 79 patients and review of the literature. J Clin Gastroenterol 1994; 18: 99-104.

18. Koch P, del Valle F, Berdel WE, Willich NA, Reers B, Hiddemann W, et al. Primary gastrointestinal non-Hodgkin's lymphoma: II. Combined surgical and conservative or conservative management only in localized gastric lymphoma-results of the prospective German Multicenter Study GIT NHL 01/92. J Clin Oncol 2001; 19: 3874-83.

19. Huang J, Jiang W, Xu R, et al. Primary gastric non-Hodgkin's lymphoma in Chinese patients: clinical characteristics and prognostic factors. BMC Cancer 2010; 10: 358

20. Binn M, Ruskoné-Fourmestraux A, Lepage E, Haioun C, Delmer A, Aegerter P, Lavergne A, Guettier C, Delchier JC. Surgical resection plus chemotherapy versus chemotherapy alone: comparison of two strategies to treat diffuse large B-cell gastric lymphoma. Ann Oncol 2003; 14: 1751-7.

21. Chihara D, Oki Y, Ine S, Kato H, Onoda H, Taji H, Kagami Y, Yamamoto K, Morishima Y. Primary gastric diffuse large B-cell Lymphoma (DLBCL): analyses of prognostic factors and value of pretreatment FDG-PET scan. Eur J Haematol 2010; 84: 493-8.

22. Wohrer S, Puspok A, Drach J, Hejna M, Chott A, Raderer M. Rituximab, cyclophosphamide, doxorubicin, vincristine and prednisone (R-CHOP) for treatment of early-stage gastric diffuse large B-cell lymphoma. Ann Oncol 2004; 15: 1086-90.

23. Leopardo D, Di Lorenzo G, De Renzo A, et al. Efficacy of Rituximab in gastric diffuse large B cell lymphoma patients. World I Gastroenterol 2010; 16: 2526-30.

24. Brugger W. Phase II Study With Rituximab and Cyclophosphamide, Doxorubicin, Vincristine, Prednisone (CHOP) in Patients With Gastric Diffuse Large B-Cell Non-Hodgkin`s Lymphoma, 2005; http://clinicaltrials.gov/ct2/show/NCT00204659. Accessed 01 August 2012

25. Sohn BS, Kim SM, Yoon DH, Kim S, Lee DH, Kim JH, Lee SW, Huh J, Suh C. The comparison between CHOP and R-CHOP in primary gastric diffuse large B cell lymphoma. Ann Hematol 2012; 91: 1731-9.

26. Zhang J, Li G, Yang H, Liu X, Cao J. Rituximab in treatment of primary gastric diffuse large B-cell lymphoma. Leuk Lymphoma 2012; 53: 2175-81.

27. Lackowska B, Gruchała A, Jaszcz-Gruchała A, Rolski J, Zemełka T, Dańda D, Ryś J. Diagnostic, predictive and prognostic verification of DNA flow cytometric measurements performed at diagnosis for non-Hodgkin's lymphoma adult patients. Pol J Pathol 2012; 62: $18-24$

\section{Address for correspondence}

\section{Mehmet Kucukoner}

Dicle University

21280 Diyarbakir, Turkey

tel. +90 (0) 4122488001

e-mail: drmehmetonko@hotmail.com

Submitted: 7.12 .2013

Accepted: $\quad 29.01 .2014$ 